\title{
Activity level recovery after acute Achilles tendon rupture surgically repaired: a series of 29 patients with a mean follow-up of 46 months
}

\author{
Richard Zayni ${ }^{1}$ \\ Raphaël Coursier ${ }^{2}$ \\ Moudasser Zakaria² \\ Jean-François Desrousseaux ${ }^{2}$ \\ Denis Cordonnier ${ }^{2}$ \\ Gilles Polveche ${ }^{2}$ \\ 1 Department of Orthopedic Surgery. Groupe Hospi- \\ talier de l'Est de la Meurthe-et-Moselle (GHEMM), \\ France \\ 2 Department of Orthopedic Surgery. Groupement \\ Hospitalier de l'Institut Catholique de Lille (GHICL), \\ France
}

Corresponding author:

Richard Zayni

Department of Orthopedic Surgery, Centre

Hospitalier de Lunéville

6 rue Girardet

B.P. 30206

54300 Lunéville, France

E-mail: richard.zayni@gmail.com

\section{Summary}

Background: Achilles tendon rupture is a common injury but its optimal management is still controversial. When decided, surgical repair can be performed by open or percutaneous techniques. Till now, there is no agreement on the ideal type of surgical management.

Purpose: To compare the outcomes of the percutaneous and open surgical treatment for acute Achilles tendon rupture and to assess the postoperative activity level recovery.

Methods: Between 2008 and 2013, 29 patients were surgically treated for acute Achilles tendon rupture in our institution. 16 patients were operated by percutaneous technique and 13 by open repair. All patients received the same postoperative rehabilitation protocol. Patients were evaluated objectively and subjectively after an average of $\mathbf{4 6}$ months (23-91).

Results: $96.6 \%$ of patients had excellent and good results according to subjective assessment. No significant difference was observed with respect to the examined clinical variables between the open and percutaneous repair groups. $20.68 \%$ of patients had minor complications related to the operation with lesser complications in the percutaneous group. $89.6 \%$ of patients resumed sport activity with an average delay of 7,7 months (4-24) and $57,7 \%$ of them resumed at a level equal or superior to their level before injury, with higher rate in the percutaneous group.

Conclusion: Percutaneous technique has similar satisfactory outcomes to open surgery in repairing acute ruptured Achilles tendon with lesser complications and higher activity level recovery rate.

Level of evidence: Retrospective comparative study. Level III.

KEY WORDS: Achilles tendon rupture, percutaneous repair, open repair.

\section{Introduction}

The rupture of the Achilles tendon (AT) is a common injury frequently encountered by orthopedic surgeon and sport medicine physician. This injury accounts for approximately $35 \%$ of all tendon tears and about $1 / 3$ of foot traumas. Almost $75 \%$ of acute ruptures occurs during recreational activities with a higher prevalence in males between 30 and 40 years of age ${ }^{1-3}$.

The incidence of this injury is increasing, particularly in non-professional athletes ${ }^{4}$. In addition to increasing sport participation, there are other factors involved in this lesion as degenerative changes in the tendon itself and the existence of a hypo-vascular region near the calcaneal insertion, which becomes prone to rupture after repetitive injuries ${ }^{1}$.

Diagnosis of acute AT rupture is usually done by history and physical examination alone, making MRI or ultrasound unnecessary for diagnosis. The patient often describes abrupt severe pain over the tendon while performing a forceful plantar flexion and, sometimes, he will feel a "pop" or have the sensation of being hit in the back of the heel. Physical examination signs suggesting AT rupture include weakness of plantar flexion and a palpable defect over the posterior calf. Another well-known diagnosis test is the "Thompson test". If there is a rupture, plantar flexion does not occur when squeezing the calf 5 .

The optimal management of this injury is still controversial $^{6,7}$. Whatever it is, this treatment should restore the original length of the tendon, minimize adhesions between it and the surrounding tissues and 
give a good cosmetic and functional result to resume full normal function and pre-injury level of activity ${ }^{8}$. This goal can be obtained by surgical, percutaneous or conservative treatment.

Decision-making regarding the treatment options relies on many factors including patient age, chronicity of the tear, medical comorbidities, patient functional demand and surgeon's preference.

Some Authors still recommend conservative management by cast immobilization, based on studies showing that non-operative treatment is clinically as efficient as surgical repair ${ }^{9,10}$. But conservative option may lead to elongation of the tendon and reduces strength of the calf muscles with a high rate of re-ruptures ranging from 10 to $30 \%{ }^{11}$. Other drawbacks of nonoperative treatment include decreased plantar flexion and endurance when compared to surgically repaired tendons ${ }^{12}$.

Consequently, surgical repair is increasingly advocated, particularly in active patients who demand early return to their pre-injury functional status. Compared to conservative management, the operative regiments present a lower rerupture rate, early functional rehabilitation with better ROM, better functional outcomes and stronger push-off with lower calf atrophy ${ }^{7,12}$.

Surgical repair can be performed by open or minimally invasive percutaneous techniques. Till now, there is no agreement which of the two techniques is ideal ${ }^{13,14}$.

Comparative studies and meta-analysis have shown that surgical treatment has higher costs and a 20 -fold higher rate of complications than conservative measures (joint stiffness, tendocutaneous adhesions, deep venous thrombosis, infection, algodystrophia and particularly the breakdown of the wound) ${ }^{15}, 16$. Given these problems, percutaneous repair, introduced by Ma and Griffith (1977), was considered as a good compromise between conservative treatment and open surgery, allowing the tendon repair in avoiding most of these complications ${ }^{8}$. Nowadays, most experts prefer the percutaneous surgical treatment to open surgery, because it is associated with a smaller number of complications and it is demonstrated to be more respectful to the biology of the tendon cicatrisation $7,8,17$. High rates of good and excellent outcomes in young adults have confirmed that new percutaneous techniques have comparable clinical effectiveness and lower complications rate than open procedures $^{6,7,14}$. Currently, many percutaneous techniques are available. In our department, we use the Tenolig ${ }^{\circledR}$ device to perform percutaneous repair for acute AT rupture. This technique has been proved as efficient as the original Ma and Griffith's technique. In a study ${ }^{17}$ comparing the two techniques, Tenolig ${ }^{\circledR}$ group shows shorter average time for operation, hospital permanence and immobilization, and it functionally stimulates the tendon healing in a short time. Functional effectiveness was the same for both techniques.

However, percutaneous technique has also complications. This includes complete and partial rerup- tures, sural neuritis, and superficial wound infection ${ }^{18}$. We present a series of patients with acute AT rupture treated surgically by open or percutaneous technique. The purpose was to report the outcomes of the surgical treatment of acute AT rupture and to compare the postoperative clinical and functional results of percutaneous versus open repair. The postoperative activity level recovery was assessed in these patients. Our hypothesis was that both treatments have similar satisfactory results in term of functional outcomes and activity level recovery.

\section{Material and methods}

\section{Patients}

We performed a retrospective cohort study to assess patients who were surgically treated for acute AT rupture in our institution from January 2008 to December 2013.

Inclusion criterion was first time closed and complete traumatic AT rupture within the last 7-10 days. Diagnosis was based on the presence of a palpable gap in the tendon, a positive Thompson test and more ankle dorsiflexion of the injured side on clinical examination. Exclusion criteria were diabetes mellitus, rheumatoid arthritis, systemic lupus erythematosus or other autoimmune disorders, chronic or recurrent tendon rupture and previous local corticosteroid treatment.

Senior experienced surgeons performed all surgeries. Because of lack of consensus on acute AT rupture management, on-call surgeon choose to perform the technique he is familiar with (open or percutaneous repair by Tenolig $\left.{ }^{\circledR}\right)$. During this period, 57 patients underwent surgery for AT rupture in our department. 33 patients $(57.9 \%)$ had percutaneous repair by Tenolig $^{\circledR}$ and 24 patients $(42.1 \%)$ had open surgical repair. 36 of the 57 patients were eligible to be included in the study.

The final clinical control was performed between October 2015 and December 2015, calling the patients for a recent follow-up. Only 29 of the 36 patients were available for this assessment: 16 patients [14 males, 2 females, mean age 34.8 (16-61)] had percutaneous repair by Tenolig ${ }^{\circledR}$ (Group $A$ ) and 13 patients [10 males, 3 females, mean age 41.2 (23-64)] had open repair (Group B).

Medical records of these 29 patients were reviewed for: age at injury time, gender, side and cause of the rupture, type of treatment, complications related to surgery (neurological, infection, DVT, PE, incidence of re-rupture) and sport-activity before the AT rupture.

The mean age of patients at the date of surgery was 37.7 years (16-64). The right tendon was affected in $44.8 \%$ of cases and the left in $55.2 \%$. 26 of the 29 patients $(89.6 \%)$ had sustained the rupture during a sports-related activity. All patients were athletes in both groups and $55.18 \%$ of patients had competitive sport activities before injury.

Table I reports the main epidemiological data of the population. 
Activity level recovery after acute Achilles tendon rupture surgically repaired: a series of 29 patients with a mean follow-up of 46 months

Table I. Epidemiological data of patients.

\begin{tabular}{|c|c|c|c|c|}
\hline Baseline data & Group A $(n=16)$ & Group B $(n=13)$ & $P$ value & Total $(n=29)$ \\
\hline $\begin{array}{l}\text { Age at surgery } \\
\text { (mean, years) (range) }\end{array}$ & $34.8(16-61)$ & $41.2(23-64)$ & 0.134 & $37.7(16-64)$ \\
\hline Gender $(M / F)$ & $14 / 2$ & $10 / 3$ & 0.798 & $24 / 5$ \\
\hline $\begin{array}{l}\text { Follow-up time } \\
\text { (mean, months) (range) }\end{array}$ & $44.3(30-81)$ & $48(23-91)$ & 0.566 & $46(23-91)$ \\
\hline Side & $7 R-9 L$ & $6 \mathrm{R}-7 \mathrm{~L}$ & 0.805 & $13 R-16 L$ \\
\hline $\begin{array}{l}\text { Delay before surgery } \\
\text { (mean, days) (range) }\end{array}$ & $1.8(1-5)$ & $6.64(1-9)$ & 0.0807 & $3.9(1-9)$ \\
\hline \multicolumn{5}{|l|}{ Sport activity } \\
\hline Competitive & 9 & 7 & & \\
\hline Badminton & 1 & 1 & & \\
\hline Basket & 1 & 2 & & 16 \\
\hline Foot & 4 & 2 & & \\
\hline Tennis & 1 & I & & \\
\hline Other & 2 & 2 & & \\
\hline Recreational & 7 & 6 & & \\
\hline Badminton & 3 & 1 & & \\
\hline Foot & 1 & 3 & & 13 \\
\hline Swimming & 2 & l & & \\
\hline Gymnastic & l & 1 & & \\
\hline Other & 1 & 1 & & \\
\hline
\end{tabular}

\section{Surgical technique}

All patients received antibiotic prophylaxis perioperatively and were operated in prone position.

Percutaneous repair was performed using Tenolig ${ }^{\circledR}$ implant (Group Fournitures Hospitalières, France). The Tenolig ${ }^{\circledR}$ device consists of $36 \mathrm{~cm}$ length and $0.85 \mathrm{~mm}$ diameter thread, onto which a $7 \mathrm{~mm}$-wide metal harpon is mounted, embedded at its distal end by a $15 \mathrm{~cm}$-long flexible triangular-tipped needle (Fig.
1A). Percutaneous surgery was performed under spinal or general anesthesia without tourniquet, on a one day-surgery basis.

The edges of AT and the rupture level were marked with a sterile pen. Then, medial and lateral $5 \mathrm{~mm}$-incisions were made about $5 \mathrm{~cm}$ proximal and distal to the rupture and enlarged with a Kelly retractor displaying the sural aponeurosis in proximal. The needle of Tenolig ${ }^{\circledR}$ device was modelled in a curve adapted

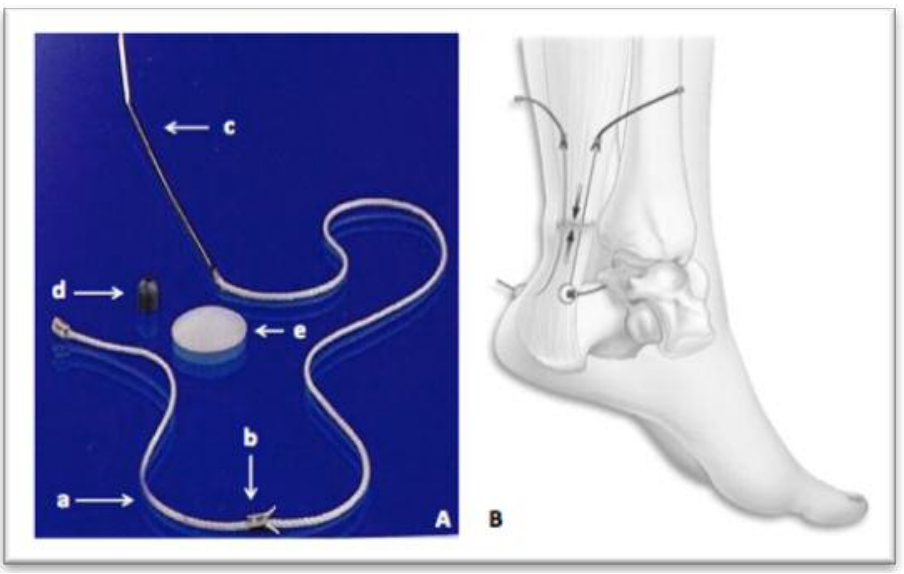

Figure 1. (Courtesy of FH - France)

A. Tenolig® implant. It is composed of:

- a thread (a) $0.85 \mathrm{~mm}$ in diameter, $36 \mathrm{~cm}$ in usable length, onto which is mounted a harpon (b) with, embedded at its distal end, a triangulartipped needle (c) with a $1.5 \mathrm{~mm}$ diameter and a length of $15 \mathrm{~cm}$, slightly curved and modelable;

- a perforated lead stopper (d) for tightening;

- a polyethylene washer (e), with a convex side allowing slight compressive pressure on skin, and a flat side.

B. Percutaneous tenosynthesis. 
to the morphology of the posterior aspect of ankle. While palpating the tendon margin by one hand, the first needle was introduced through the lateral or medial proximal incision, passed through the two stumps of the tendon and recovered from the distal retromalleolar incision on the same side. Same procedure was then repeated on the other side. By pulling progressively the threads distally, the two metal anchors were introduced and applied on sural aponeurosis through proximal incisions. A special attention was made while introducing anchor in the lateral proximal incision, to avoid sural nerve entrapment. Holding the two wires ending with the needles, the surgeon flexed the ankle to maximal equinus, bringing the two stamps closer together, then plastic washers were inserted with the convex side against the skin and fixed by the lead stoppers. At the end, anterior cast splint was applied keeping the ankle in gravity equinus.

For Tenolig ${ }^{\circledR}$ surgical technique (Fig. 1B) see also ${ }^{17,} 19$. Concerning the open repair, surgery was performed under spinal or general anesthesia and a tourniquet was always used. An end-to-end suturing was done using Krackow et al. ${ }^{20}$ technique, through a posteromedial incision done approximately $10 \mathrm{~cm}$ long and 1 $\mathrm{cm}$ medial to the tendon's edge, using a non-absorbable No. 2 suture. By the end, the paratenon was closed with absorbable No. 3.0 suture, a meticulous hemostasis was performed to avoid hematoma formation, and skin incision was closed using interrupted non-absorbable No. 2.0 stitches. An attention was made when manipulating the skin edges while closing to avoid necrotic cutaneous lesions ${ }^{16}$. After surgery, an anterior cast splint was applied keeping the ankle in gravity equinus. The skin sutures were removed within 15 days after surgery.

\section{Post-operative care and rehabilitation}

Anti-thrombotic treatment with low molecular weight heparin was administered to all patients until resumption of normal ambulation.

Postoperative rehabilitation was almost the same for both groups. The anterior splint maintaining the ankle in plantar flexion was kept for 21 days, during which weight bearing was forbidden. Mobilization was started immediately at $\mathrm{d} 3$ and consisted in passive and assisted active plantar flexion with active dorsal flexion no more than $90^{\circ}$. Since the third week, partial weight bearing with a walking orthosis and a heel lift maintaining a $30^{\circ}$ plantar flexion was started during 3 additional weeks in both groups

In group A patients, the Tenolig ${ }^{\circledR}$ sutures were removed around 6 weeks after surgery, and full weight bearing was then started, with stepwise decreases in the height of the heel lift at 2-week intervals to get $0^{\circ}$ by the end of the third month.

In group B patients, orthosis was removed 6 weeks after surgery. Then, patients begun total weight bearing while decreasing the height of the heel lift to get $0^{\circ}$ by the end of 3 months.

In both groups, triceps-strengthening exercises and resumption of sports training were started after 3 months.

\section{Follow-up and clinical evaluation}

Patients were reviewed at an average of 46 months (23-91) after the index surgery for objective and subjective evaluation.

Clinical examination and functional evaluation were performed by a single experimented examiner. Ankle range of motion was assessed bilaterally by goniometry, with $0^{\circ}$ corresponding to the neutral orthogonal ankle position. The maximum calf circumference was measured in both the affected and the contralateral leg by using a commercially available tape measurer, $25 \mathrm{~cm}$ proximal to the medial malleolus. Maximum calf circumference is proved correlated with triceps muscle volume 21

The AT width on both sides was measured $5 \mathrm{~cm}$ above his calcaneus insertion. By palpation, edges of the tendon at this level were felt and marked then distance between edges was measured by tape measurer. A detailed neurological examination focused on sural nerve was done.

Patients were also requested to perform two tasks more specific to a repair of an AT rupture, namely, the ability to stand on tiptoes for $30 \mathrm{~s}$ and to perform ten times the single-limp hopping 22

Subjective evaluation was done using the Achilles tendon total rupture score (ATRS). This score is a self-administered instrument with proved clinical utility in evaluating the outcomes, related to symptoms and physical activity, after treatment in patients with a total AT rupture ${ }^{23}$. We also used the ankle and hindfoot score of the American Foot and Ankle Society (AOFAS) to evaluate the subjective satisfaction at the most recent follow-up. Results were rated as excellent (100-90 points), good (89-80 points), fair (79-70), or poor $(<70)$ according to Kitaoka et al. ${ }^{22}$.

At the final follow-up, long-term complications (such as rerupture, infection, sural neuroma, Achilles tendinitis, scare adhesion), patients' return to work and activity level recovery were recorded. Any modifications or changes of previous sports activity after treatment were also noted.

The study protocol was approved by the local ethical committee and meets the standards and lows of MLT Journal as described by Padulo et al. ${ }^{24}$.

\section{Statistical analysis}

The data were recorded in a Microsoft Excel sheet (Microsoft Corporation, USA) and analyzed using SPSS 24 statistical software (SPSS Inc., Chicago, IL, USA). Statistical analysis was performed with the Student's $t$ test for comparisons between the groups. Pearson test was used for correlation study. $P$ values $<0.05$ were considered significant.

\section{Results}

The mean follow-up (FU) of patients was 46 months (23-91). No significant difference was found in pre-injury baseline data between the two groups of patients (Table I).

The highest incidence of rupture is found in 31-40 
Activity level recovery after acute Achilles tendon rupture surgically repaired: a series of 29 patients with a mean follow-up of 46 months

year-old patients (41.4\%), followed by $41-50$ years old $(24.2 \%)$. Six of the 29 reviewed patients $(20.68 \%)$ had complications related to the operation. Two $(12.5 \%)$ minor complications were identified in Group A (1 patient had early superficial wound infection controlled by local wound caring and another patient had a hypoesthesia in the sural nerve dermatome at the last follow-up). In Group B, 4 complications (30.76\%) were identified ( 2 patients had superficial wound infection treated by local wound caring, 1 patient presented wound healing delay and another patient presented scar adherence found at the last clinical control). No major complication such as DVT or PE was noted in the two groups. None of the patients with complications had a systemic disease, previous AT symptoms or injections with corticosteroids.

The mean delay before surgery was 3.9 days (1-9). No correlation was found between this delay and AOFAS score $(r=0.0585, p=0.763)$.

In Group A, the mean Tenolig ${ }^{\circledR}$ removal delay was 52.3 days (35-80). No correlation was found between this delay and AOFAS score of the Group A patients $(r=0.2772, p=0.3173)$

\section{Clinical objective assessment}

At the most recent FU, all patients were able to stand on their toe tips for more than $30 \mathrm{~s}$ and all were able to perform single-limb hopping for ten times.

\section{Calf diameter}

Side-to-side triceps diameter average difference was found equal to $2.25 \mathrm{~cm}(0-12): 2.06 \mathrm{~cm} \mathrm{(0-6)}$ in Group A and $2.5 \mathrm{~cm} \mathrm{(0-12)} \mathrm{in} \mathrm{Group} \mathrm{B;} p=0.628$.

At the last FU, the maximum calf circumference was not significantly decreased on the operated leg compared to the uninjured side; $p=0.063$.

\section{ROM}

Difference in dorsal flexion between the intact and the operated ankle was found $0.48^{\circ}\left(-8^{\circ}-6^{\circ}\right)$ in average with no significant inter-side difference; $p=$ 0.746 .

Dorsiflexion side-to-side difference was respectively $0.43^{\circ}\left(-8^{\circ}-6^{\circ}\right)$ and $0.53^{\circ}\left(-4^{\circ}-4^{\circ}\right)$ in Group $A$ and Group B; $p=0.936$.

Difference in plantar flexion between the intact and the operated ankle was found $3.3^{\circ}\left(-8^{\circ}-17^{\circ}\right)$. No significant inter-side difference was found; $p=0.322$.

Plantar flexion side-to-side difference was respectively $3.62^{\circ}\left(-8^{\circ}-12^{\circ}\right)$ and $2.92^{\circ}\left(-6^{\circ}-17^{\circ}\right)$ in Group A and Group B; $p=0.789$.

\section{Width of AT}

The operated AT was found enlarged of a mean of $0.94 \mathrm{~cm}(0.5-2)$ if compared to the intact side $(p<$ $0.05)$. This enlargement was $0.93 \mathrm{~cm}(0.5-2)$ in Group A and $0.96 \mathrm{~cm}(0.5-1.5)$ in Group B. No significant inter-group difference was found; $p=0.871$.

Table II shows side-to-side difference of all clinical variables assessed at last FU.

\section{Subjective assessment and functional scores}

The mean overall SRTA score was 84.34 (27-100): $83.125(27-100)$ in Group A and $85.84(54-100)$ in Group B; $p=0.681$.

The mean overall AOFAS score was 94.55 points (71-100): 94.37 (71-100) for Group A and 94.76 (81$100)$ for group $\mathrm{B} ; p=0.890$.

$96.6 \%$ of reviewed patients had excellent or good results (Tab. III).

Table II. Side-to-side difference of all clinical variables assessed at last FU.

\begin{tabular}{lllll}
\hline Side-to-side difference concerning: & Group A & Group B & Total & $p$ \\
\hline Plantar flexion (degree) (interval) & $3.62(-8-12)$ & $2.92(-6-17)$ & $3.3(-8-17)$ & 0.789 \\
Dorsal flexion (degree) (interval) & $0.43(-8-6)$ & $0.53(-4-4)$ & $0.48(-8-6)$ & 0.936 \\
Calf diameter (cm) (interval) & $2.06(0-6)$ & $2.5(0-12)$ & $2.25(0-12)$ & 0.628 \\
Tendon width (cm) (interval) & $0.93(0.5-2)$ & $0.96(0.5-1.5)$ & $0.94(0.5-2)$ & 0.871 \\
\hline
\end{tabular}

Table III. SRTA \& AOFAS scores.

\begin{tabular}{lcccccc}
\hline & \multicolumn{2}{c}{ Group A } & \multicolumn{2}{c}{ Group B } & \multicolumn{2}{c}{ Total } \\
\hline Scores/grading & SRTA & AOFAS & SRTA & AOFAS & SRTA & AOFAS \\
Excellent $(>90)$ & 6 & 10 & 8 & 8 & 14 & 18 \\
Good $(75-90)$ & 8 & 5 & 5 & 5 & 13 & 10 \\
Poor $(65-75)$ & $/$ & 1 & $/$ & $/$ & $/$ & 1 \\
Unsatisfactory $(<65)$ & 2 & $/$ & $/$ & 2 & $/$ \\
\hline
\end{tabular}


Table IV. Functional results of percutaneous and open repair of acute rupture of Achilles tendon.

\begin{tabular}{llll}
\hline Variable & Group A ( $\mathbf{n = 1 6 )}$ & Group B $(\mathbf{n = 1 3})$ & $\boldsymbol{p}$ \\
\hline Time to return to work (months) (interval) & $5.75(1-24)$ & $4.6(2-12)$ & 0.631 \\
Time to return to sport (months) (interval) & $7.2(5-24)$ & $8.16(4-18)$ & 0.759 \\
AOFAS (points) (interval) & $94.37(71-100)$ & $94.76(81-100)$ & 0.890 \\
ATRS (points) (interval) & $83.125(27-100)$ & $85.84(54-100)$ & 0.681 \\
Satisfaction & $93.75 \%$ & $100 \%$ & 0.761 \\
\hline
\end{tabular}

$96.5 \%$ of patients graded their satisfaction good or excellent $(93.75 \%$ in Group A and $100 \%$ in Group B). One patient has permanent residual pain, 7 have mild occasional pain and 21 patients $(72 \%)$ were painless.

\section{Sport and activity resumption}

Three patients no longer wanted indulge in sports, particularly the sport during which the injury occurred, mostly for fear of re-injury. Two patients were of Group A and 1 of Group B. Satisfaction and subjective scores of these 3 patients were comparable to the remaining patients.

At the last FU, of the 26 patients $(89.6 \%)$ who resumed sport activity: 15 patients $(57.7 \%)$ resumed at a level equal or superior to their level before injury: 9/14 (64.3\%) in Group A and 6/12 (50\%) in Group B. The mean time to return to previous activities was 7.7 months (4-24): 7.2 months (5-24) for Group A and 8.16 months (4-18) for Group B; $p=0.759$.

The mean time to return to work was 7.68 months (124): 5,75 months (1-24) in Group $A$ and 4.6 months (2-12) in Group B; $p=0.631$.

Functional results of percutaneous and open repair are resumed in Table IV.

\section{Discussion}

Our study has mainly found that surgical repair of acute AT rupture (open and percutaneous) was efficient and both techniques had similar clinical and functional outcomes. At the last follow-up, almost $90 \%$ of patients returned to sport activity. $58 \%$ of them resumed sport at a level similar or superior to the pre-injury level, after a mean delay of almost eight months. This rate was higher in the percutaneous repair group with $2 / 3$ of patients able to resume at same or higher level.

Many Authors studied the return to sport after AT rupture surgically repaired. In Guillo series ${ }^{25}, 81 \%$ had returned to their pre-operative sport at same or higher level. For Chiu et al. ${ }^{26}$, endoscopically-assisted percutaneous AT repair allowed $95 \%$ of patients returning to their previous level of sporting activity after 6 months. In Hsu series ${ }^{27}, 88 \%$ of patients (included professional athletes) returned to their previous sportive activities after an interval of 3 months.

The same level activity recovery rate in our series was inferior if compared to other studies ${ }^{25-27}$. Any of athlete patients reviewed was professional, which is usual in this type of injury where most of victims are recreational athletes. Therefore, return to sport activity in a same level was not mandatory or a goal in itself for these patients. In addition, time to return to sport was relatively longer compared to other series ${ }^{26-28}$. This long delay of sport resuming may be due to the fact that patients were not high level professional athletes and preferred having total remission before returning to sport because of apprehension and fear of re-rupture. Saxena et al. ${ }^{29}$ proposed a solution that could resolve this apprehension problem, predicting patient ability to return to previous activities by using standardized parameters as single-legged concentric strengthening, ROM and muscle volume.

In 1977, Ma and Griffith ${ }^{8}$ described a percutaneous technique for the repair of acute AT rupture that became popular thereafter ${ }^{19}, 30$. Previous evidence suggests that open compared to percutaneous repair has lower rate of re-rupture, higher rate of return to pre-injury activity level, higher complication rates, namely infection, and almost the double $\operatorname{cost}^{25,31}$. No significant differences were found between open and percutaneous repair in terms of muscle volume, tendon's length, and days off-work ${ }^{21}$. In a randomized study, Lim et al. ${ }^{32}$ found a higher rate of wound infections ( $21 \mathrm{vs}$ $9 \%$ ) in the open repair group with comparable re-ruptures rate and functional results in both groups. Similarly, another study ${ }^{33}$ reported equally good results in terms of low risk of re-rupture and complications with satisfactory clinical and functional outcomes for percutaneous repair. Our results are concordant with these conclusions except the rate of return to pre-injury activity level found slightly higher in percutaneous group. Complication rate, mainly infection and wound dehiscence, was found higher in patients with open repair (22 vs $6 \%$ in percutaneous group).

Sural nerve injury and neuroma formation is known to be a frequent complication of percutaneous acute AT repair ranging from 13 to $60 \%{ }^{18,34}$. Using novel techniques such as local anesthesia, endoscopic or ultrasound control has reduced the risk of this complication $25,26,35$. In our series, sural nerve injury was observed in $6.25 \%$ of patients in the percutaneous treatment only. To reduce this risk, we controlled sural nerve by direct observation through the proximal lateral incision. 
Activity level recovery after acute Achilles tendon rupture surgically repaired: a series of 29 patients with a mean follow-up of 46 months

Clinical evaluation of AT width, sural triceps trophism and tibio-tarsal joint ROM at the last FU demonstrated no significant differences between the two groups. We obtained satisfactory results in term of ankle ROM by these two techniques and calf diameter difference between the operated and non-operated legs was not significant. In addition, no significant difference concerning the tendon width was found between the two groups, contrarily to other studies ${ }^{14,36}$. These results can be explained by the early mobilization that could help in muscle recovery, remodeling of the tendon and against articular stiffness. Early mobilization was possible in both groups. Despite Tenolig ${ }^{\circledR}$ is a percutaneous device, it cannot be considered as a real suture. But it remains a minimally invasive procedure because it avoids exposing the rupture site and devascularization of the tendon. It works in bringing blindly the tendon stamps closer together. Because of the metallic anchor placed on aponeurosis, the traction exerted on the proximal portion of the tendon is made along its longitudinal axis. The hauling effect on the proximal stump approaches the distal stump reproducing similar physiological results with respect to the forces that normally ensure the proper function of the tendon and its subsequent healing. The very strong wire used in this device allows early mobilization of the tendon ${ }^{17}$.

Because of lack of consensus, many surgeons still manage acute AT rupture by open repair advocating their attitude by the higher rerupture rate and neural complication in the percutaneous treatment. As mentioned above, these complications can be avoided by adopting new assisted surgical techniques ${ }^{26,35}$ with efficient rehabilitation protocols encouraging early mobilization and weight bearing. It is time to shift to percutaneous repair as a new cost-effective ${ }^{31}$ gold standard for surgical management of AT rupture. Nowadays, most current evidence shows that percutaneous surgery results in a lower rate of complications than open surgery, with a comparable functional outcomes $^{37,38}$ (Tab. V).

Table V. Main conclusions of most recent studies comparing open and percutaneous AT repair.

\begin{tabular}{|c|c|c|c|c|}
\hline Authors & Year & Type of study & $\begin{array}{l}\text { Number of } \\
\text { patients }\end{array}$ & Main conclusions \\
\hline Hsu et al. ${ }^{27}$ & 2015 & Retrospective & $\begin{array}{l}\text { Percutaneous: } 101 \\
\text { Open: } 169\end{array}$ & $\begin{array}{l}\text {-Higher rate of return to prior activity in percutaneous } \\
\text { group } \\
\text {-No difference in term of complications between the } 2 \\
\text { groups }\end{array}$ \\
\hline $\begin{array}{l}\text { Karabinas } \\
\text { et al. }{ }^{28}\end{array}$ & 2014 & Retrospective & $\begin{array}{l}\text { Percutaneous: } 19 \\
\text { Open: } 15\end{array}$ & $\begin{array}{l}\text {-No difference in term of wound healing, complications, } \\
\text { ankle ROM, patients' return to work, activity level, } \\
\text { weight-bearing, and subjective assessment of treatment } \\
\text {-Better cosmetic appearance in the percutaneous group }\end{array}$ \\
\hline $\begin{array}{l}\text { Carmont } \\
\text { et al. }{ }^{31}\end{array}$ & 2013 & Retrospective & $\begin{array}{l}\text { Percutaneous: } 49 \\
\text { Open: } 35\end{array}$ & $\begin{array}{l}\text {-Comparable clinical outcomes and complications rates } \\
\text { - Lesser theatre occupation and hospital stay in } \\
\text { percutaneous group } \\
\text {-Percutaneous repair is a cost-effective surgical } \\
\text { management of AT rupture }\end{array}$ \\
\hline $\begin{array}{l}\text { Henriquez } \\
\text { et al. }{ }^{39}\end{array}$ & 2012 & Retrospective & $\begin{array}{l}\text { Percutaneous: } 17 \\
\text { Open: } 15\end{array}$ & $\begin{array}{l}\text {-Similar outcomes in term of plantar flexion strength, } \\
\text { ROM, calf and ankle perimeter, and single heel raising } \\
\text { test } \\
\text {-Mean 'return to work' time longer in open repair group } \\
\text {-Mean scar length greater in open repair group } \\
\text {-Better cosmetic appearance, lower rate of wound } \\
\text { complications and no apparent increase in the risk of } \\
\text { rerupture in percutaneous group }\end{array}$ \\
\hline $\begin{array}{l}\text { Gigante } \\
\text { et al. }{ }^{14}\end{array}$ & 2008 & $\begin{array}{l}\text { Prospective } \\
\text { randomized }\end{array}$ & 40 patients & $\begin{array}{l}\text {-Comparable medium-term results } \\
\text {-No difference in SF-12 questionnaire, ultrasound and } \\
\text { isokinetic assessment } \\
\text {-No differences in clinical parameters except for ankle } \\
\text { circumference, found greater in percutaneous group } \\
\left(T_{e n o l i g}^{\circledR}\right) \\
\text {-Percutaneous repair reduces cutaneous complications } \\
\text { and operation times, enables faster recovery, enhancing } \\
\text { overall patient compliance. It is preferable to open repair } \\
\text { in managing subcutaneous ruptures of AT in non- } \\
\text { professional sports practicing adults }\end{array}$ \\
\hline
\end{tabular}

to be continued 
Continue from Table V

\begin{tabular}{|c|c|c|c|c|}
\hline $\begin{array}{l}\text { Cretnik } \\
\text { et al. }{ }^{34}\end{array}$ & 2005 & Retrospective & $\begin{array}{l}\text { Percutaneous: } 132 \\
\text { Open: } 105\end{array}$ & $\begin{array}{l}\text { - Comparable functional results, with significantly lower } \\
\text { rate of complication in percutaneous repair } \\
\text {-No difference in subjective assessment (AOFAS \& Holz } \\
\text { score) }\end{array}$ \\
\hline $\begin{array}{l}\text { Haji } \\
\text { et } \mathrm{al}^{40}\end{array}$ & 2004 & Retrospective & $\begin{array}{l}\text { Percutaneous: } 38 \\
\text { Open: } 70\end{array}$ & $\begin{array}{l}\text {-No difference in term of ROM, stiffness, power and } \\
\text { complications } \\
\text {-Percutaneous repair, in experienced hands, is a suitable } \\
\text { alternative to traditional open techniques }\end{array}$ \\
\hline
\end{tabular}

Table $\mathrm{V}$ shows the main conclusions of most recent studies that compare open and percutaneous AT repair.

This study had many weakness points as its retrospective type, the small number of patients and the relatively short follow-up time. In addition, a part of patients was unreachable for the last control. This may cause a bias in the assessment of complications rate at medium-term follow-up.

Also, there is heterogeneity in surgical management because different surgeons performed surgery. That may expose to an inter-surgeon variability in procedure achieving. Our department is a university medical center and during meeting staffs, we try to unify surgical techniques and indications. Finally, no objective assessment was performed to study the tendon healing or the functional recovery as ultrasound or isokinetic evaluation.

\section{Conclusion}

In conclusion, the present study showed that both open and percutaneous techniques are similarly effective in repairing the ruptured AT and result in nearly total restoration of clinical and functional patterns if complications can be avoided. Medium-term results were substantially comparable with an unified early rehabilitation protocol. Percutaneous repair had lesser complications with higher rate of returning to previous sport activity level.

A part of patients is still unable to return to previous level of sport activity, sometimes because of apprehension. Further studies with longer follow-up are required to evaluate long-term outcomes and to better understand the factors involved in the activity recovery.

\section{Acknowledgements}

Authors would like to thank Audrey Carton and AnneSophie Coulon, for their precious collaboration in achieving this study.

\section{Conflict of interest}

The Authors have no financial or personal relationships with other people or organizations that could inappropriately influence their work.

\section{References}

1. Jozsa L, Kvist M, Balint BJ, Reffy A. The role of recreational sport activity in Achilles tendon rupture. A clinical, pathoanatomical and sociological study of 292 cases. Am J Sports Med. 1989;17:338-343.

2. Maffulli N, Ajis A, Longo UG, Denaro V. Chronic rupture of tendo Achillis. Foot Ankle Clin. 2007;12:583-596.

3. Suchak A, Bostick G, Reid D, Blitz S, Jomha N. The incidence of Achilles tendon ruptures in Edmonton, Canada. Foot Ankle Int. 2005;26:932-936.

4. Nyyssönen $T$, Lüthje $P$, Kröger $H$. The increasing incidence and difference in sex distribution of Achilles tendon rupture in Finland in 1987-1999. Scand J Surg. 2008;97(3):272.

5. Thompson TC. A test for rupture of the tendo achillis. Acta Orthop Scand. 1962;32:461-465.

6. Goren D, Ayalon M, Nyska M. Isokinetic strength and endurance after percutaneous and open surgical repair of Achilles tendon ruptures. Foot Ankle Int. 2005;26:286-290.

7. Khan RJ, Fick D, Keogh A, Crawford J, Brammar T, Parker M Treatment of acute Achilles tendon ruptures. A metaanalysis of randomized, controlled trials. J Bone Joint Surg Am. 2005;87:2202-2210.

8. Ma GWC, Griffith TG. Percutaneous repair of acute closed ruptured Achilles tendon: a new technique. Clin Orthop. 1977;128:247-255

9. Ingvar J, Tagil M, Eneroth M. Nonoperative treatment of Achilles tendon rupture: 196 consecutive patients with a 7\% rerupture rate. Acta Orthop. 2005;76:597-601.

10. Riaz JK, Dan F, Angus K, et al. Treatment of Acute Achilles tendon ruptures. A meta-analysis of randomized, controlled trials. J Bone Joint Surg Am. 2005;87:2202-2210.

11. Strauss EJ, Ishak C, Jazrawi L, Sherman O, Rosen J. Operative treatment of acute Achilles tendon ruptures: an institutional review of clinical outcomes. Injury. 2007;38(7):832-838.

12. Kocher MS, Bishop J, Marshall R, Briggs KK, Hawkins R. Operative versus nonoperative management of acute Achilles tendon rupture. Am J Sports Med. 2002;30:783-788.

13. Horstmann T, Lukas C, Merk J, Brauner T, Mundermann A. Deficits 10-years after Achilles tendon repair. Int J Sports Med. 2012;33(6):474-479.

14. Gigante A, Moschini A, Verdenelli A, Del Torto M, Ulisse S, de Palma L. Open versus percutaneous repair in the treatment of acute Achilles tendon rupture: a randomized prospective study. Knee Surg Sports Traumatol Arthrosc. 2008;16:204209.

15. Maffulli N, Longo UG, Oliva F, Ronga M, Denaro V. Minimally invasive surgery of the Achilles tendon. Orthop Clin North Am. 2009 Oct; 40 (4):491-498.

16. Saxena A, Maffulli N, Nguyen A, Li A. Wound Complications with Achilles tendon surgery: report on 219 cases. J Am Podiatr Med Assoc. 2008;98(2):95-101.

17. Taglialavoro G, Biz C, Mastrangelo G, Aldegheri R. The repair 
of the Achilles tendon rupture: comparison of two percutaneous techniques. Strat Traum Limb Recon. 2011;6:147-154.

18. Maes R, Copin G, Averous $\mathrm{C}$. Is percutaneous repair of the Achilles tendon a safe technique? A study of 124 cases. Acta Orthop Belg. 2006;72:179e83.

19. Delponte P, Potier L, De Poulpiquet P, Buisson P. Traitement des ruptures sous-cutanees du tendon d'Achille par tenorraphie percutanée. Rev Chir Orthop. 1992;78:404-407.

20. Krackow KA, Thomas SC, Jones LC. A new stitch for ligamenttendon fixation. Brief note. J Bone Joint Surg Am. 1986;68 (5): 764-766.

21. Claudio R, Patrick V, Caroline P, et al. Long-term outcomes of muscle volume and Achilles tendon length after Achilles tendon ruptures. Knee Surg Sports Traumatol Arthrosc. 2013; 21:1369-1377.

22. Kitaoka HB, Alexander IJ, Adelaar RS, Nunley JA, Myerson MS, Sanders M. Clinical rating systems for the ankle-hindfoot, midfoot, hallux, and lesser toes. Foot Ankle Int. 1994;15(7): 349-353.

23. Nilsson-Helander K, Thomee R, Silbernagel KG, et al. The Achilles tendon Total Rupture Score (ATRS): development and validation. Am J Sports Med. 2007;35:421e6.

24. Padulo J, Oliva F, Frizziero A, Maffulli N. Muscles, Ligaments and Tendons Journal - Basic principles and recommendations in clinical and field science research: 2016 Update. MLTJ. 2016; 6(1):1-5.

25. Guillo S, Del Buono A, Dias M, Denaro V, Maffulli N. Percutaneous repair of acute ruptures of the tendo Achillis. Surgeon. 2013;11(1):14-19.

26. Chiu CH, Yeh WL, Tsai MC, Chang SS, Hsu KY, Chan YS. Endoscopy-assisted percutaneous repair of acute Achilles tendon tears. Foot Ankle Int. 2013;34(8):1168-1176.

27. Hsu AR, Jones CP, Cohen BE, Davis WH, Ellington JK, Anderson RB. Clinical Outcomes and Complications of Percutaneous Achilles Repair System Versus Open Technique for Acute Achilles Tendon Ruptures. Foot Ankle Int. 2015;36 (11):1279-1286

28. Karabinas P, Benetos IS, Lampropoulou-Adamidou K, et al. Percutaneous versus open repair of acute Achilles tendon ruptures. Eur J Orthop Surg Traumatol. 2014;24:607-613.
29. Saxena A, Ewen B, Maffulli N. Rehabilitation of the operated Achilles tendon: parameters for success. J Foot Ankle Surg. 2011;50(1):37-40.

30. Soubeyrand M, Serra-Tosio G, Campagna R, Molina V, Sitbon $\mathrm{P}$, Biau DJ. Intraoperative ultrasonography during percutaneous Achilles tendon repair. Foot Ankle Int. 2010;31(12): 1069-1074.

31. Carmont MR, Heaver C, Pradhan A, Mei-Dan O, Gravare Silbernagel K. Surgical repair of the ruptured Achilles tendon: the cost-effectiveness of open versus percutaneous repair. Knee Surg Sports Traumatol Arthrosc. 2013;21(6):1361-1368.

32. Lim J, Dalal R, Waseem M. Percutaneous vs. open repair of the ruptured Achilles tendon-a prospective randomized controlled study. Foot Ankle Int. 2001;22(7):559-568.

33. Ceccarelli F, Berti L, Giuriati L, Romagnoli M, Giannini S. Percutaneous and minimally invasive techniques of Achilles tendon repair. Clin Orthop Relat Res. 2007;458:188-193.

34. Cretnik A, Kosanovic M, Smrkolj V. Percutaneous versus open repair of the ruptured Achilles tendon: a comparative study. Am J Sports Med. 2005;33:1369-1379.

35. Lacoste S, Féron JM, Cherrier B. Percutaneous Tenolig repair under intra-operative ultrasonography guidance in acute Achilles tendon rupture. Orthopaedics \& Traumatology: Surgery \& Research. 2014;100:925-930.

36. Bradley JP, Tibone JE. Percutaneous and open surgical repairs of Achilles tendon ruptures. A comparative study. Am J Sport Med. 1990;18:188-195.

37. Del Buono A, Volpin A, Maffulli N. Minimally invasive versus open surgery for acute Achilles tendon rupture: a systematic review. Br Med Bull. 2014;109:45-54.

38. Ververidis AN, Kalifis KG, Touzopoulos P, Drosos G, Tilkeridis $\mathrm{KE}$, Kazakos KI. Percutaneous repair of the Achilles tendon rupture in athletic population. Journal of orthopaedics. 2016; 13:57-61.

39. Henríquez H, Muñoz R, Carcuro G, Bastías C. Is percutaneous repair better than open repair in acute Achilles tendon rupture? Clin Orthop Relat Res. 2012;470(4):998-1003.

40. Haji A, Sahai A, Symes A, Vyas JK. Percutaneous versus open tendon achillis repair. Foot Ankle Int. 2004;25(4):215218. 